This article was published as: Archive of Applied Mechanics 89 (1), 91-103, 2019.

DOI: https://doi.org/10.1007/s00419-018-1450-2 


\section{Rheological properties of magnetic biogels}

\section{A. Zubarev, ${ }^{1}$ A.B. Bonhome-Espinosa, ${ }^{2,3}$ M. Alaminos, ${ }^{3,4}$ J.D.G. Duran, ${ }^{2,3}$ M.T. Lopez- Lopez $^{2,3}$}

${ }^{1}$ Department of Theoretical and Mathematical Physics, Ural Federal University, Ekaterinburg, Russia

${ }^{2}$ Department of Applied Physics, University of Granada, Granada, Spain.

${ }^{3}$ Instituto de Investigación Biosanitaria ibs.GRANADA, Granada, Spain

${ }^{4}$ Department of Histology (Tissue Engineering Group), University of Granada, Granada, Spain

\section{Introduction}

Magnetic polymers (gels and elastomers) are composite materials consisting of nano-sized magnetic particles embedded into a gel matrix. Combination of rich set of physical properties of the polymer and magnetic systems is very perspective for many progressive industrial, bioengineer and biomedical applications. In part, these composites are used as magnetocontrollable dampers, vibration and shock absorbers [1-7 ]. Hydrogels with biocompatible magnetic particles are very promising for various biomedical technologies - for drug delivery and biosensors [8-14]; constriction of soft actuators and artificial muscles $[2,15]$; for regenerative medicine and tissue engineering [16-35]. An overview on the synthesis of magnetic hydrogels and their biomedical applications can be found in [23].

Nonmagnetic biocompatible hydrogels are actively used in bioengineering and clinical medicine as scaffolds for manufacturing, curing and regeneration of biological tissues [36,37]. The scaffolds, prepared of magnetic hydrogels, have several advantages in front of their nonmagnetic analogies. First, magnetic particles allow in-vivo visualization of the growing tissue, by using the magnetic resonance imagining [31]. Second, as some experiments demonstrate, the particles stimulate proliferation of the tissue cells $[6,38,39]$. Third, in vivo applications, magnetic particles functionalized with the agents of the tissue growth, can be attracted to the magnetic scaffold, providing intensification of this process [6,25,31]. Fourth, invivo, magnetic hydrogels can be injected into the place of the tissue regeneration in a liquid state, with the help of a syringe, i.e. by the microinvasive way. The gel, up to its curing, can be fixed in this place by using magnetic field of necessary configuration. Next, rheological properties and behavior of these magnetic implants can be controlled by the external field $[33,40,41]$.

The above-mentioned advantages of the magnetic hydrogels in front of the nonmagnetic ones, from the viewpoint of their biomedical applications, attract considerable interest of researches and bioengineers to these systems. Many works have been devoted to the manufacturing, study and practical use of magnetic biological hydrogels. However, in spite of these efforts, fundamental features of many physical properties and behaviour of these materials are still unclear. Furthermore, there are still many challenges from the viewpoint of the design of 
magnetic hydrogels. First, most magnetic hydrogels, reported in the literature, are based on polymers cross-linked covalently. These hydrogels are usually very robust, but at the same time too rigid to allow tunability of their biomechanical properties at a large degree by application of magnetic fields. Furthermore, the pore size of covalent hydrogels is usually reduced, which hinders the diffusion of nutrients and oxygen and the removal of waste in biomedical applications. On the other hand, physically cross-linked hydrogels present a large pore size, adequate for applications, but unfortunately they are usually too weak from the biomechanical viewpoint and they lack of the required microstructure - they are usually microscopically inhomogeneous. In view of this, present and near-future efforts in the field of magnetic hydrogels should focus on the preparation of hydrogels with adequate internal order, biomechanical properties and with magnetic field-responsive behaviour.

In this paper we review our recent progress on the field of the experimental study and modeling of the rheological properties of magnetic biogels. Our experimental system consists of fibrin networks embedded with magnetite nanoparticles and swelled by water-based solutions. Fibrin is a natural protein involved in the clotting of blood and consequently it has inherent biocompability and biodegradability. As magnetic phase we used commercial magnetite nanoparticles.

\section{Materials and methods}

Ethics statement. This study was approved by the Ethics Committee of the University of Granada, Granada, Spain.

For the preparation of the magnetic biogels we used frozen human plasma of healthy blood donors (provided by the Granada Biobank of the Andalusian Regional Government) as source of the fibrin polymer that constituted the polymer network. As magnetic phase we used commercial MagP-OH nanoparticles (nanoMyP®, Spain). These particles consisted of a core of magnetite $(90 \% \mathrm{v} / \mathrm{v})$ coated by a layer of polymer $(10 \% \mathrm{v} / \mathrm{v})$ provided with $\mathrm{OH}^{-}$functionalization. They were spherical in shape and had a mean hydrodynamic diameter of $110 \mathrm{~nm}$, as measured by dynamic light scattering with a Zetasizer instrument (Malvern instruments, USA). For additional details of the magnetic particles see [42]. For some biogels we also used type VII agarose (a polysaccharide polymer material with a molecular weight of approx. 120,000 g/mol, supplied by Sigma-Aldrich Química SA, Madrid, Spain) to strengthen the polymer matrix to achieve higher concentrations of magnetic particles within the gel. Note that the addition of agarose to fibrin gels produces more consistent and easier-to handle biomaterials [43]. In addition, we used in the preparation of gels Phosphate Buffered Saline (PBS) with $\mathrm{MgCl}_{2}$ and $\mathrm{CaCl}_{2}$, liquid, sterilefiltered, and suitable for cell culture, supplied by Sigma-Aldrich (Spain), $\mathrm{CaCl}_{2}$ powder (calcium chloride dehydrate, Sigma Ultra, minimum 99.0\%) provided by Sigma-Aldrich, tranexamic acid (Amchafibrin $500 \mathrm{mg}$ tablets, ROTTAPHARM, S.L.) and Milli-Q water (Millipore).

\section{Synthesis of the biogels}

For the preparation of the magnetic biogels we used protocols reported in details in previous papers $[33,42]$. Briefly, for the preparation of $5 \mathrm{~mL}$ of biogel, we poured $3.8 \mathrm{~mL}$ of human plasma in a culture dish and afterwards added to them $875 \mu \mathrm{L}$ of a suspension of magnetic particles and agarose in PBS, $75 \mu \mathrm{L}$ of a solution of tranexamic acid at a concentration of $0.1 \mathrm{~g} / \mathrm{mL}$, and $250 \mu \mathrm{L}$ of $2 \% \mathrm{CaCl}_{2}$ in aqueous solution. The concentration of particles and agarose in PBS were adjusted 
in order to obtain the desired concentrations in the final gels. It should be noted that we worked with only two concentrations of agarose: 0 and $0.1 \mathrm{~g} / \mathrm{mL}$ of agarose with respect to the volume of the final gel -we will refer to the gels with $0 \mathrm{~g} / \mathrm{mL}$ of agarose as fibrin gels, whereas we will refer to the gels with $0.1 \mathrm{~g} / \mathrm{mL}$ of agarose as fibrin-agarose gels. Note at this point that in the absence of agarose (fibrin gels) we failed to prepare homogeneous gels for particle concentrations above 0.4 vol. $\%$. Because of this, at higher concentrations of particles we added agarose at a concentration of $0.1 \mathrm{~g} / \mathrm{mL}$ (fibrin-agarose gels), which gave consistence to the gels and allowed to obtain homogeneous gels. The tranexamic acid is an antifibrinolytic agent that prevents degradation of the scaffold. As for the $\mathrm{CaCl}_{2}$, it activates the fibrin polymerization process [44]. Immediately after the addition of the $\mathrm{CaCl}_{2}$ solution, the polymerization started. During the whole polymerization process ( 24 hours) we kept the mixtures under cell culture conditions $\left(37^{\circ} \mathrm{C}\right.$ with $5 \% \mathrm{CO}_{2}$ ). During the first $5 \mathrm{~min}$ of gelation we applied to the gelling samples a vertical magnetic field with the help of a coil connected to a DC power supply. We investigated different intensities of the applied magnetic field $(0,16.2,32.4$ and $48.6 \mathrm{kA} / \mathrm{m})$. After the first 2 hours of gelation we added $5 \mathrm{~mL}$ of PBS to the already gelled gels to prevent their dehydration. After 24 hours we took the biogels out of the culture dishes and characterized them immediately afterwards. The biogels had the shape and size of the culture dishes where they were prepared: disk-like shape of $35 \mathrm{~mm}$ of diameter.

\section{Microstructure of the biogels}

For the characterization of the microstructure of the biogels we used scanning electron microscopy (SEM). For this aim we prepared the samples by the technique of Critical Point Drying (CPD), which allows conservation of the original structure. For details see [33,42].

\section{Rheological characterization of the biogels}

We characterized the mechanical properties of the biogels with a Haake MARS III (Thermo Fisher Scientific, Waltham, MA, USA) controlled stress rheometer at $37^{\circ} \mathrm{C}$, using parallel plate geometry of $35 \mathrm{~mm}$ of diameter with rough surfaces made of titanium (sensor P35Ti L, Thermo Fisher Scientific, Waltham, MA, USA). For measurements we proceeded as it follows. First we took the biogel out of the culture dish and placed it in the measuring system, descending the upper (rotating) plate until perfect contact with the sample was reached. Then, we characterized the samples under constant values of the shear strain. We increased (linearly spaced) the values of the shear strain and monitored the resulting values of the shear stress. We carried out measurements under the application of magnetic fields of different intensity in the range $0-26 \mathrm{kA} / \mathrm{m}$. For this purpose we used a coil connected to a DC power supply, with the axis of the coil vertically aligned (i.e., the resulting field was vertical). For measurements under a magnetic field, we connected the field 1 min before the start of the measurement and maintained it until the measurement was over.

\section{Experimental results}

\section{Microstructure of the biogels}


Figure 1 shows images taken by scanning electron microscopy (SEM) of nonmagnetic gels (Figure 1a) and magnetic gels (both gelled in absence and presence of applied magnetic field). It can be observed that the nonmagnetic gel (see Figure 1a) had fibrin fibers distributed forming a relatively dense porous structure. Furthermore, we can see that the structure had not any junction, except for the homogeneously distributed links between couples of fibers. On the other hand, for magnetic gel with 0.2 vol. \% MagP-OH in absence of a magnetic field (see Figure 1b), we observe a much opener structure, with knots formed presumably by clusters of magnetic nanoparticles and polymer material. These knots very likely performed as cross-linkers between long polymer fibers, making possible to maintain an opener structure, avoiding the collapse of the fibers. For magnetic gel with 0.2 vol.\% $\mathrm{MagP}-\mathrm{OH}$ gelled in presence of a magnetic field (see Figures 1c and 1d), we can see a heterogeneous structure, consisting of roughly parallel regions with dense concentration of polymer fibers, separated by some regions with low density of fibrin fibers -this is more evident at lower magnification (Figure 1d) and for fibrin-agarose gels containing much higher concentration of magnetic particles (Figure 1h). This fiber distribution justifies a higher swelling degree of this kind of magnetic gels in comparison with nonmagnetic gels, although the porosity seems to be lower than for magnetic gels gelled in the absence of magnetic field. Note that no chains of magnetic particles are evidenced in Figures 1c, 1d, and 1e, in spite of the fact that gelation was initiated (first 5 minutes) under the presence of an applied magnetic field for these gels. This can be explained by considering that the magnetic particles were attached to the fibrinogen in the initial mixture, before the application of the magnetic field. Thus, application of the magnetic field resulted in the partial alignment of the fibrin fibers along the magnetic field direction.
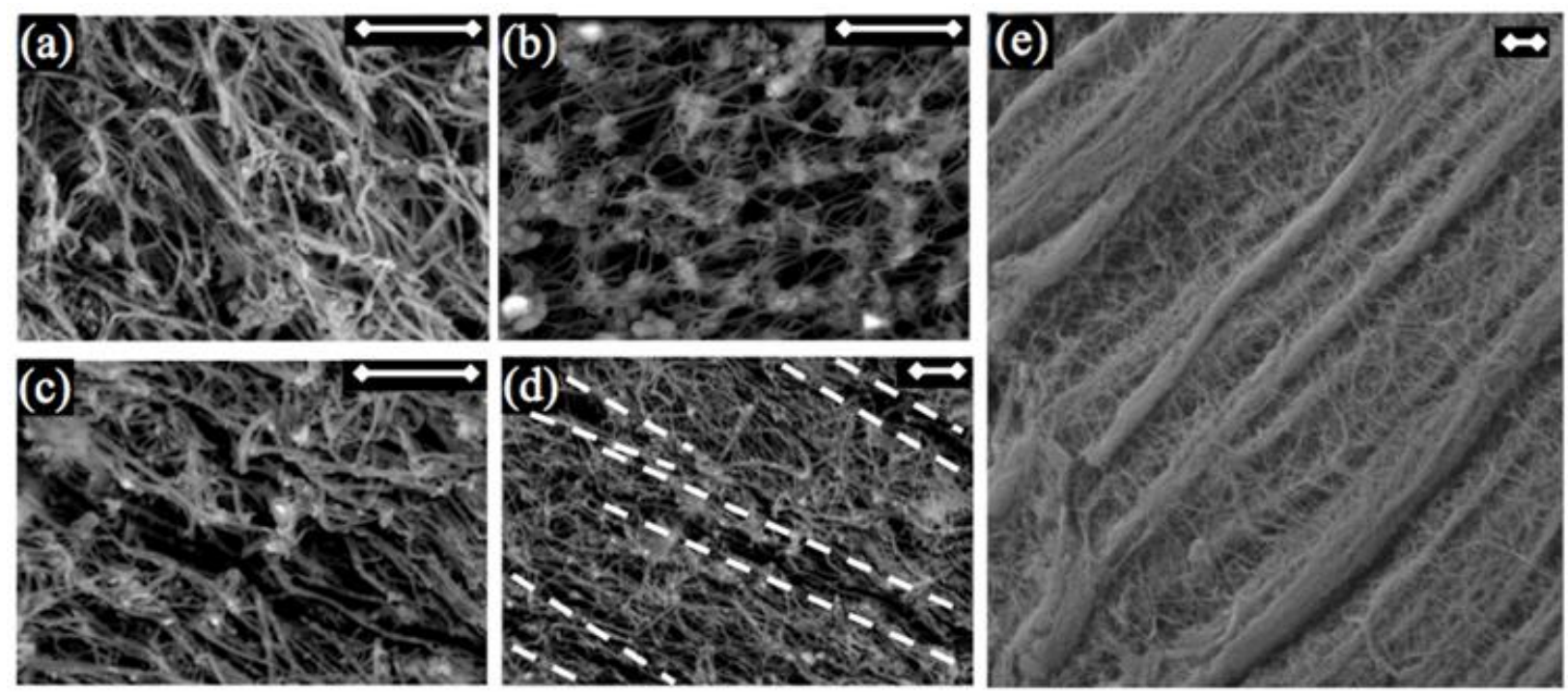

Figure 1. Images of scanning electron microscopy of gels. (a) Fibrin gel; (b) fibrin gel with 0.2 vol.\% of magnetic nanoparticles gelled in absence of a magnetic field; (c) and (d) fibrin gel with 0.2 vol.\% of magnetic nanoparticles gelled in presence of a $48.6 \mathrm{kA} / \mathrm{m}$ magnetic field; (e) fibrin-alginate gel with 3.5 vol. $\%$ of magnetic nanoparticles gelled in presence of a $48.6 \mathrm{kA} / \mathrm{m}$ magnetic field. Dashed lines in (d) delimit the regions with low concentration of fibrin fibers. Bar length: 5 microns. (a) and (b) are reproduced from [42] by permission of The Royal Society of Chemistry; (e) is adapted from [33]. 
Let us finally mention that similar microstructures to these of Figures $1 \mathrm{~b}$ to $1 \mathrm{~d}$ were observed for magnetic fibrin gels with concentration of magnetic particles smaller than 0.5 vol.\% (pictures not shown here). On the other hand, for larger particle concentrations the amount of clusters within the fibrin gels largely dominated over connecting polymer fibers (pictures not shown here for brevity), which is consistent with failure of formation of macroscopically homogeneous gels, in agreement with macroscopic observations of fragile gels with lumpy appearance. To overcome this problem we added agarose at a concentration of $0.1 \mathrm{~g} / \mathrm{mL}$ for concentrations of particles above 0.4 vol. $\%$. Agarose gave consistence to the resulting fibrin-agarose gels and allows obtaining homogeneous magnetic gels with higher concentrations (Figure 1e).

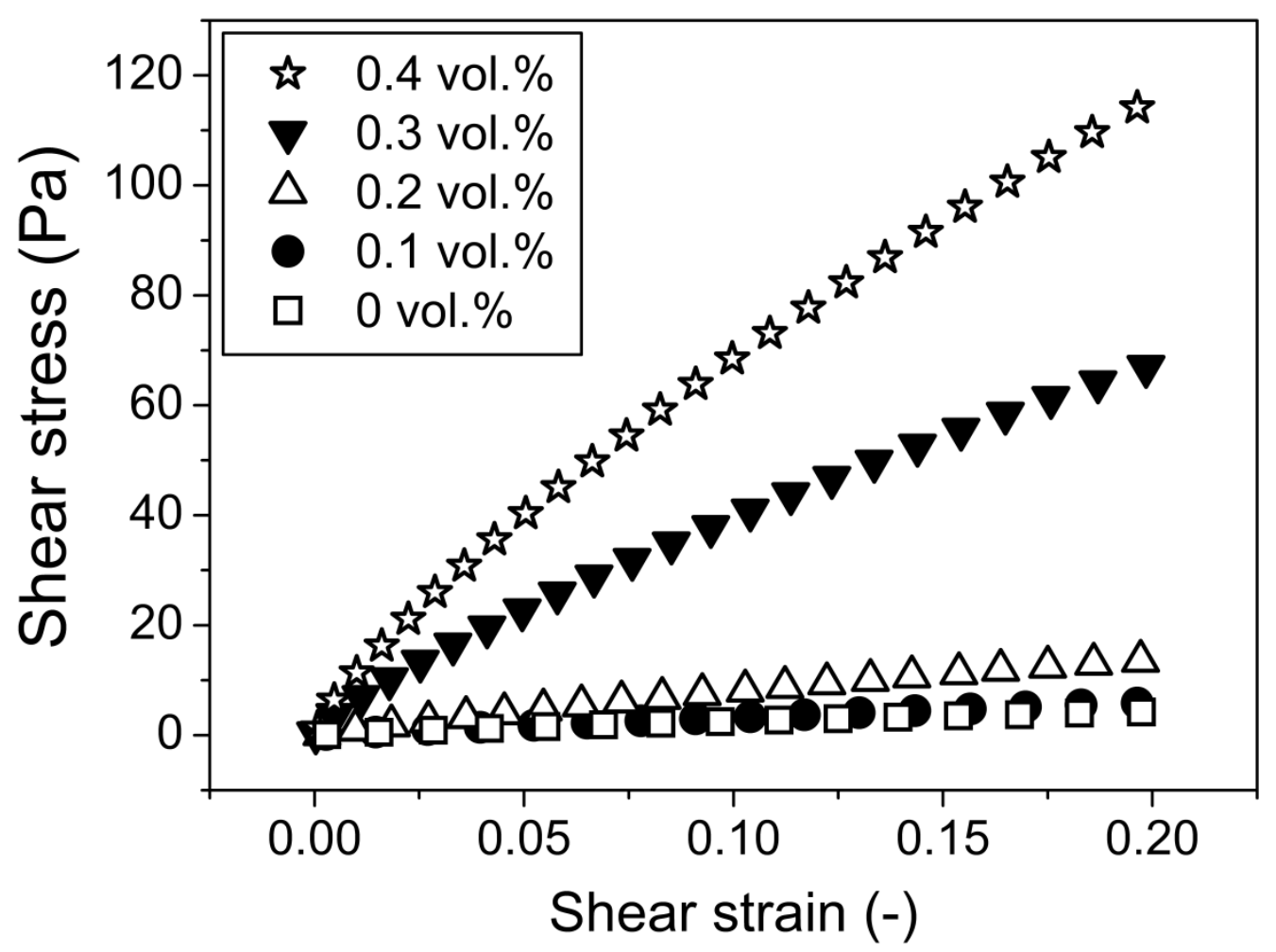

Figure 2. Shear stress obtained under imposed values of the shear strain for magnetic fibrin gels gelled in the absence of magnetic field. Volume concentration of magnetic nanoparticles in the biogels is indicated.

\section{Rheological characterization of the biogels}

In order to focus on the interaction between the nanoparticles and the fibrin polymer, first we studied fibrin biogels (without agarose addition). The behavior of the biogels under constant shear strain was characterized by measuring of the shear stress resulting from imposed values of shear strain increased stepwisely. Curves of shear stress vs. shear strain were concave in all cases (i.e., the slope of the curves presented a tendency to decrease as the shear strain increased), as typical for an elastic material -see Figure 2 as an example (similar trends were obtained for biogels gelled in the presence of applied magnetic field). 

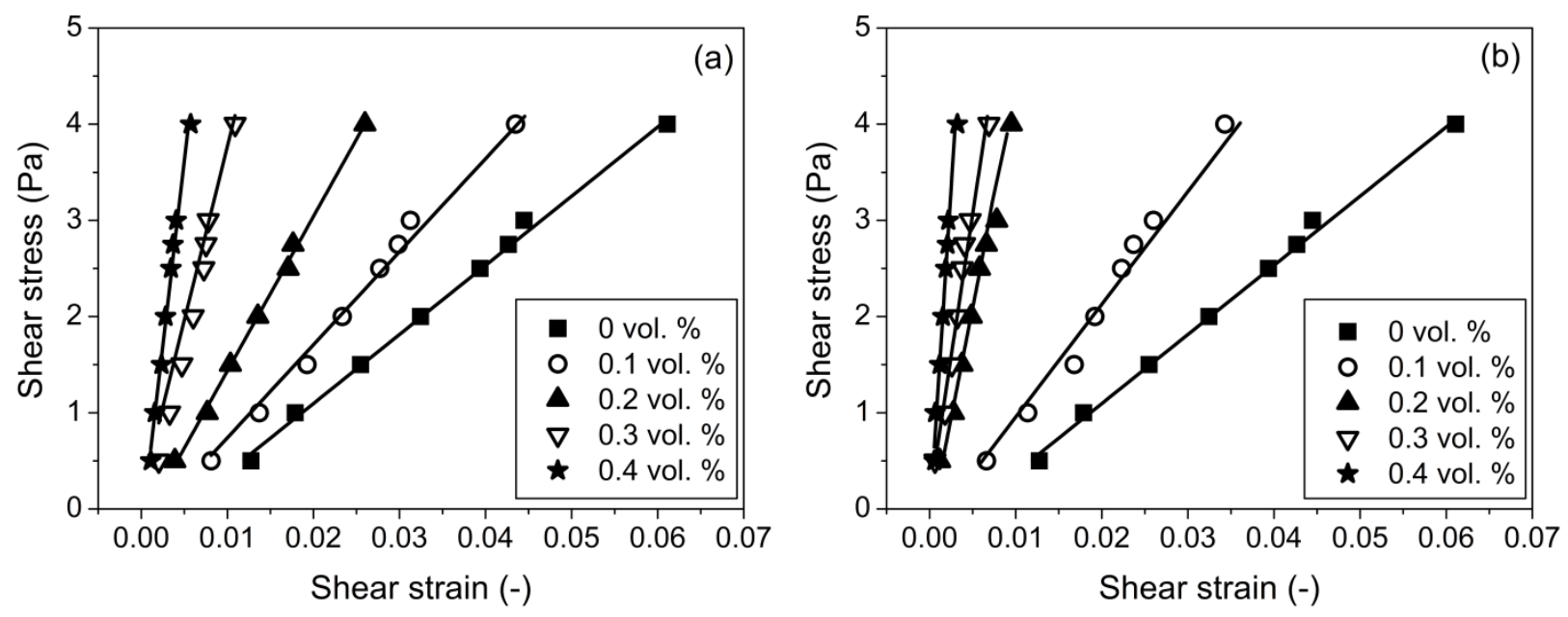

Figure 3. Shear stress obtained under imposed values of the shear strain for magnetic fibrin biogels -only the initial (linear) part of the curves is shown. Volume concentration of magnetic nanoparticles (MNP) in biogels is indicated. Symbols represent experimental data and the continuous lines the best fits to straight lines. (a) Biogels gelled in the absence of magnetic field. (b) Biogels gelled for the first 5 minutes under the application of a magnetic field of $48.6 \mathrm{kA} / \mathrm{m}$. (a) is reproduced from [42] by permission of The Royal Society of Chemistry.

At low shear strain the curves could be approximated to straight lines, with a characteristic slope (Figure 3). This initial slope of the shear stress vs. shear strain curves is the rigidity modulus, which is one of the quantities that are used to measure the stiffness of a material. For magnetic fibrin biogels, the rigidity modulus increased faster than linearly with the concentration of magnetic nanoparticles up to a magnetic particle content of 0.4 vol.\% (Figure 4). As mentioned above, for concentration of magnetic nanoparticles higher than 0.4 vol.\% the magnetic fibrin biogels were not homogeneous from the macroscopic point of view and, consequently, their rheological characterization was not carried out. 


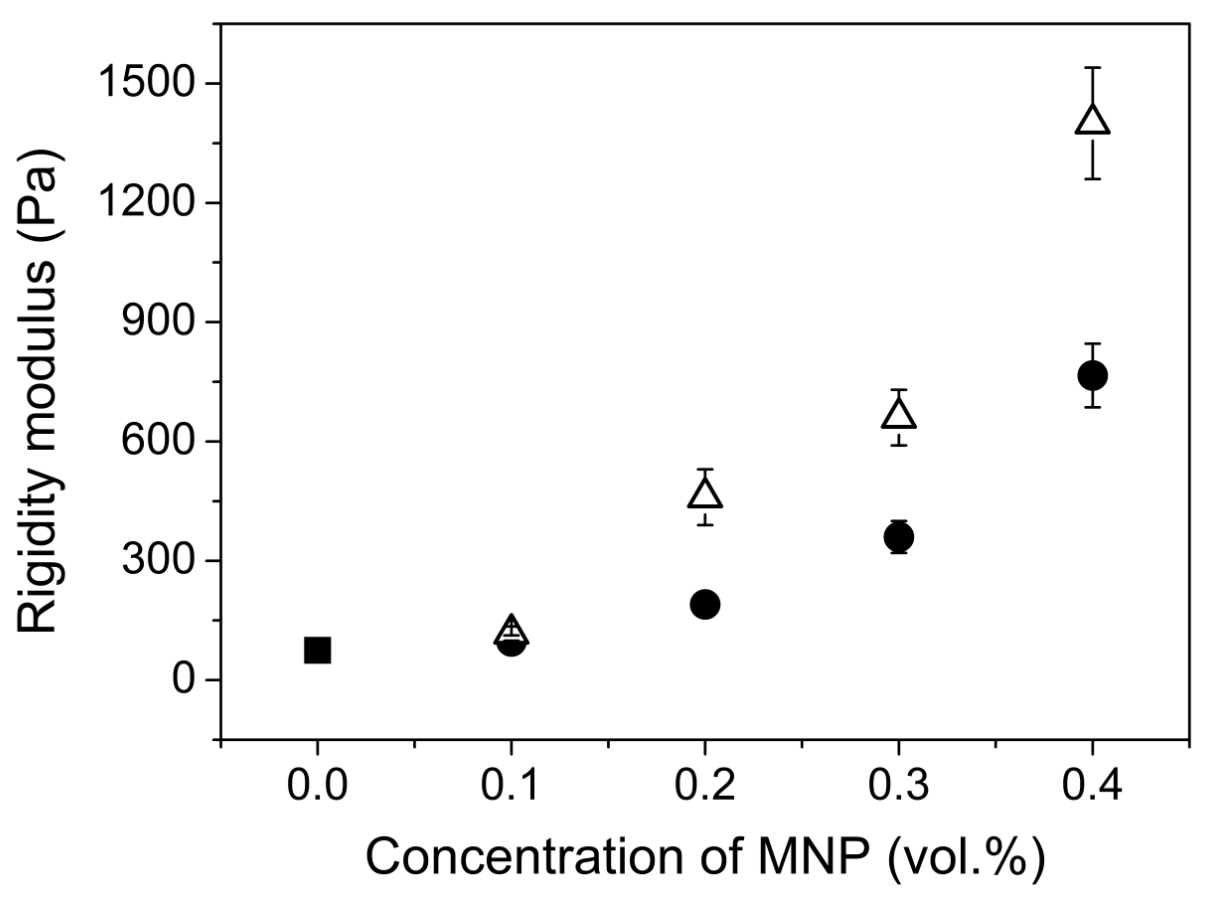

Figure 4. Rigidity modulus of magnetic fibrin biogels for increasing concentration of magnetic nanoparticles (MNP). Volume concentration of MNP is indicated. $\mathbf{m}$ : Nonmagnetic gel.

-: Ferrogels gelled in the absence of magnetic field. $\Delta$ : Ferrogels gelled for the first 5 minutes under the application of a magnetic field of $48.6 \mathrm{kA} / \mathrm{m}$. The modulus of the nonmagnetic gel is $75 \mathrm{~Pa}$.

Concerning the comparison between magnetic biogels gelled in presence of magnetic field and magnetic biogels gelled in absence of magnetic field, the former presented a faster increase of the rigidity modulus with the magnetic particle content (Figure 4). In both cases (biogels gelled in the presence or absence of applied field) the rigidity modulus increased very fast with concentration of magnetic particles. For the concentration 0.4 vol. $\%$, the composite modulus is more than ten times greater than the modulus of the pure fibrin gel. Note that the classical Einstein formula for the mechanical modulus of dilute disperse systems predicts that this increase must be about 1 per cent. Thus the disagreement between these experiments and the classical theories of the composite materials is about three orders of magnitude. This means that these theories, in principle, cannot describe the rheological effects in the biological ferrogels. 


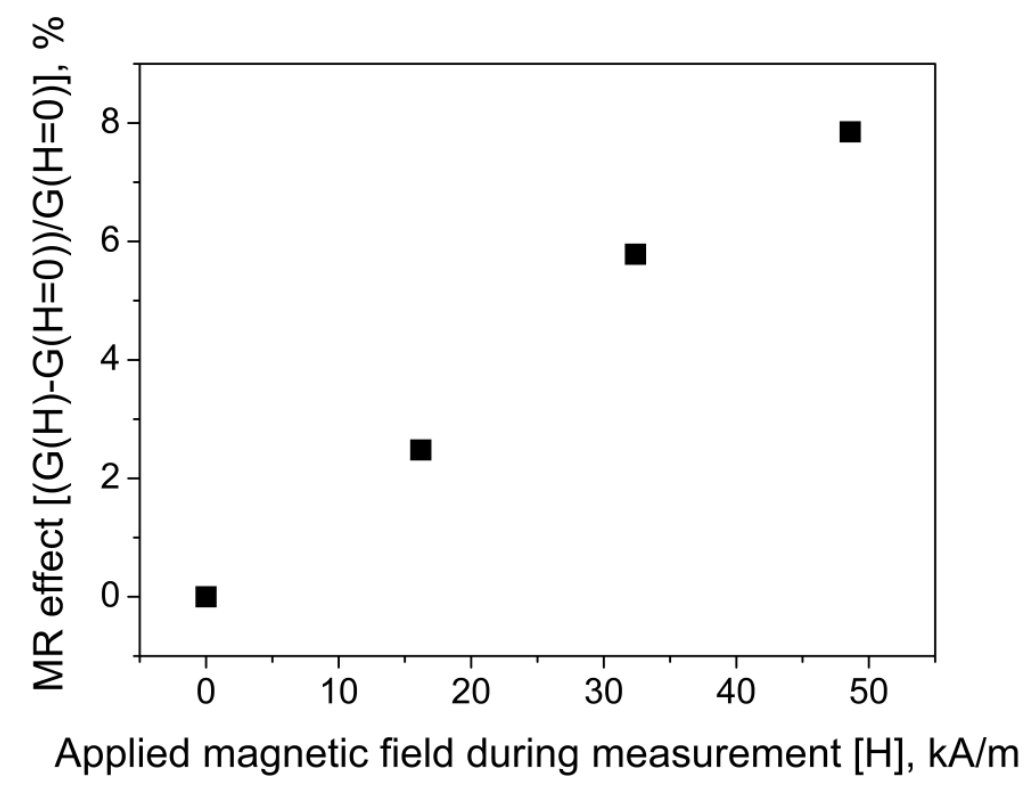

Figure 5. Magnetorheological (MR) effect: i.e., effect of an applied magnetic field, $H$, on the rigidity modulus $(\mathrm{G})$ of magnetic fibrin-agarose biogels containing 3.5 vol.\% of magnetic nanoparticles. The $x$ axis represents de magnetic field applied during rheological measurements. Data shown in the Figure are for biogels gelled under an applied magnetic field of $48.6 \mathrm{kA} / \mathrm{m}$ of strength -similar results were obtained for other magnetic field strengths.

\section{Magnetorheological characterization of the biogels}

With regards to the rheological behavior under an applied magnetic field, we checked that the effect of the applied magnetic field on the rheological properties (magnetorheological effect) was negligible for magnetic fibrin biogels containing up to 0.4 vol. $\%$ of magnetic particles. This result is reasonable in view of the minute amount of magnetic nanoparticles. As mentioned above, for concentration of magnetic nanoparticles higher than 0.4 vol. $\%$ the resulting magnetic fibrin biogels were inhomogeneous and broke easily under manipulation. In order to prepare biogels with higher concentration of magnetic particles, we prepared biogels with addition of agarose at a concentration of $0.1 \mathrm{~g} / \mathrm{mL}$. This concentration of agarose allows to obtain fibrin-agarose hydrogels with proper consistency to admit concentrations of magnetic nanoparticles of about 3-4 vol.\% without breakage. For these magnetic fibrin-agarose hydrogels, we analyzed the effect of an applied magnetic field during measurements. We fixed the concentration of magnetic particles within the fibrin-agarose biogel at 3.5 vol.\%. Results of the magnetorheological (MR) effect in the shear modulus, $\mathrm{G}$, quantified as the different between the rigidity modulus in the presence of field $(\mathrm{G}(\mathrm{H}))$, minus the rigidity modulus in the absence of field $(\mathrm{G}(\mathrm{H}=0))$, divided by $\mathrm{G}(\mathrm{H}=0)$, are plotted in Figure 5. As observed, there is a weak, linear enhancement of the rigidity modulus with the applied magnetic field during measurement (MR effect), which for the highest applied field reached about $8 \%$.

\section{Theoretical approach.}

The fibrin macromolecule presents a thread with complicated internal structure. The typical length of the thread is of the order of several tens of microns, and diameter of the order of $100 \mathrm{~nm}$ (see 
Figure 1a). The fibrin fibers were formed by polymerization of fibrinogen (from blood plasma) in the presence of $\mathrm{Ca}^{2+}$ ions. Fibrinogen is a glycoprotein consisting of three different chains $(\alpha, \beta$, and $\gamma$ ), linked to each other by disulfide bonds and forming a central $\mathrm{E}$ domain (negatively charged), two identical peripheral D domains (negatively charged), and two $\alpha \mathrm{C}$ domains (positively charged) [45-47]. MagP-OH nanoparticles had negative surface charge as a consequence of $\mathrm{OH}^{-}$functionalization. As a consequence, fibrinogen molecules were expected to anchor on the MagP-OH nanoparticles. A particle, adsorb on a fibrin molecule, because of electrostatic interaction, can anchor another molecule. This situation is illustrated in Fig.6.

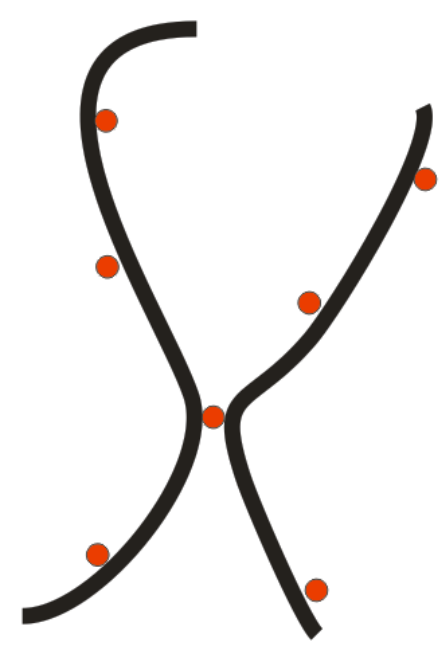

Figure 6. Scetch of mutual attraction of the fibrin threads (black curves) due to adsorbed particle (red dots)

Thus the particles, adsorbed on the fibrins, provoke indirect (through the particles) attraction between the macromolecular threads. This attraction can induce the fibrin phase condensation. The domains of the dense phase are observed in experiments and are seen in Figs.1b (light dots) for the hydrogel cured without the field and in Figs 1c,d,e for the gel, cured under the field. It is important to note that the pure fibrin hydrogel, without the particles, does not experience the phase separation and takes place in mezoscopically uniform state (Fig 1a).

\section{Gel cured without field.}

Let us discuss now the internal physical cause of formation of the macroscopic elastic properties for the hydrogel, polymerized without the field. This is well known that the final stage of phase separations in liquid media includes recondensation of the discreet nuclei into one bulk domain of new phase [48]. To explain the stable state of the composite hydrogel with the discreet dense knots (Figs.1b), we suppose that initially the supersaturation of the particlefibrinogen system was high and, consequently, this system experienced a sudden (instantaneous) nucleation. The polymer-particles knots in this scenario are similar to the drop of dense phase at the gas-liquid phase transition. Due to the fast rate of the nucleation, the supersaturation decreased quickly, and the rate of the "drop" growth diminished very fast. Furthermore, polymerization of fibrins out of the "drops" should also contribute to the interruption of the drop evolution. Thus, in agreement with the observations, we can suppose the instantaneous stop of 
the growth of the knots right after their appearance. In more details analysis of the systems with the discreet nuclei of the dense polymer-particle phase is given in [42].

One can see from the Fig. $1 \mathrm{~b}$ that each dense nuclear of the fibrin dense phase cross-links the fibrin macromolecules. The number of the links per the nuclear is determined by many factors. Its empirical value, estimated from the photos of the systems is of the order of 10. Thus, appearance of the polymer-particle knots increases the number of the subchain in the cured gel. It follows from the classical theory of elasticity of the polymer nets $[49,50]$ that the net elastic modulus is proportional to the number of the subchains per the volume unity of the system. Therefore appearance of the knots, as additional centers of the fibrin cross-linkage, increases the gel rigidity.

The shear elastic modulus $G$ of the fibrin ferrogels has been estimated in [42] on the basis of this concept. The result is presented in the form

$G=G_{0}+A \varphi_{0}^{2}$

Here $G_{0}$ is the modulus of the pure hydrogel without the particles, $\varphi_{0}$ is the total volume concentration of the particles in the ferrogels, $A \approx 4.3 \cdot 10^{7} \mathrm{~Pa}$ is a semi-empirical parameter, estimated in [42].

Comparison of the theoretical and experimental results are shown in Fig.7.

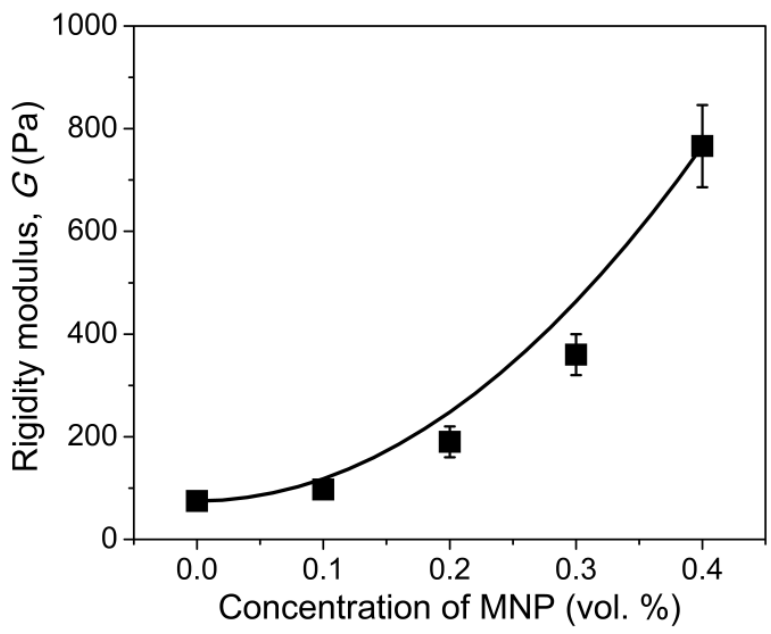

Figure 7. Rigidity modulus of magnetic hydrogels for increasing concentrations of magnetic nanoparticles. Squares represent the experimental data. The continuous line (-) represents the theoretical prediction according to eq. (1), for $G_{0}=75 \mathrm{~Pa}$. Reprinted from [42] by permission of The Royal Society of Chemistry.

The agreement with the experimental results and the formula given by eq. (1) is quite reasonable. Thus, at least from the semiquantitative point of view, our model explains the experimental trend, and can be considered as a robust background for the further investigations.

Gel cured under the field action.

If, during polymerization, the hydrogles is placed in external magnetic field, the nuclear of the fibrin-particles dense phase are being elongated in the field direction and amalgamate under the 
forces of the magnetic attraction. As a consequence, the column-like aggregates, elongated in the field direction, appear in the system. Our observations show that these columns percolate the measuring chamber with the ferrogels and the intercolumn space is much less concentrated that the columns are. The situation is schematically illustrated in Fig.8.

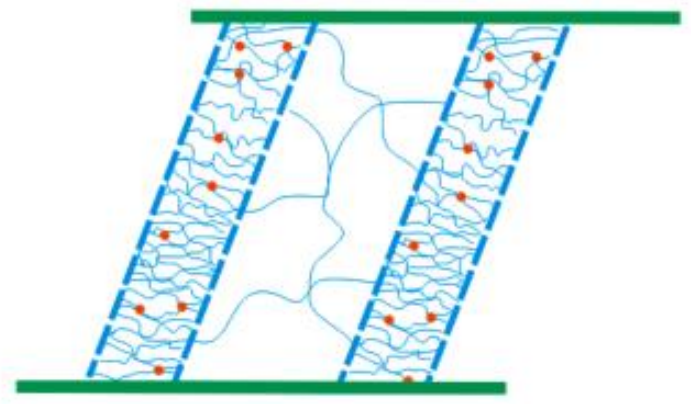

Figure 8. Scetch of the hydrogel with the dense column-like aggregates, percolating the chamber with the ferrogel. Red dot are the particles.

If the aggregates are linked, as the rest of the gel, with the walls of the gap, the total shear modulus $G$ of the system can be estimated according to the "rule of mixtures" [51] :

$G=G_{d} \Phi+G_{e}(1-\Phi)=\left(G_{d}-G_{e}\right) \Phi+G_{e} \approx G_{d} \Phi+G_{e}$

Here $\Phi$ is volume concentration of the domains, $G_{d}$ and $G_{e}$ are the modulus of the gel in the dense domains (aggregates) and out of them (in the dilute environment) respectively. We take into account that the strong inequality $G_{d} \gg G_{e}$ must be held.

In the composites with low concentration of the domains $(\Phi \ll 1)$ the modulus $G_{e}$ of the interdomain medium must be approximately equal to the modulus $G_{0}$ of the pure gel. When $\Phi$ is comparable with 1 (say, $\Phi \approx 0.2$ and more), the inequality $G_{d} \Phi \gg G_{e}$ is fulfilled (since $G_{d}$ is much greater than $G_{e}$ ), therefore $G_{e}$ does not play a significant role in eq. (1). That is why, in the further estimates, we can use the approximation $G_{e} \approx G_{0}$ without a serious mistake.

Taking into account that concentration of the fibrins outside the columns is much less than that inside them, in the first approximation the aggregates volume concentration $\Phi$ can be estimated as:

$\Phi \approx \frac{\psi_{0}}{\psi_{d}}$

Here $\psi_{0}$ and $\psi_{d}$ are the total volume concentration of the fibrin in the composite and its concentration inside the domain respectively.

Let us suppose again that inside the aggregates the fibrin cross-linkage takes place, mainly, according to the scenario illustrated in Fig. 7. In the other words, we suppose that, inside the domains, the particles play a main role as the centers of this cross-linkage.

The concentration $v$ of these cross-linking "ties" in the domain, roughly, can be estimated as: $v \sim \varphi_{d} \psi_{d}^{2}$

where $\varphi_{d}$ is volume concentration of the particles in the cylindrical domain of the dense phase. Indeed, the tie presents a cluster, consisting of one particle and two fibrins. Concentration $v$ is 
proportional to the probability that the particle and two fibrins are situated closely to each other. This leads directly to the ratio (4).

Of course, the real situation can be much more complicated, however, in a first approximation, we can explain the main physical features of the system by supposing the simplest structure of the tie - 1 particle and 2 fibrin fragments .

This seems logically to suppose that the particles are more or less homogeneously adsorbed on the fibrins - each fibrin thread contains the same number of particles as the other ones. In the framework of this assumption, we get the scaling relation

$\varphi_{d} \sim \varphi_{0} / \Phi=\varphi_{0} \frac{\psi_{d}}{\psi_{0}}$

Here $\varphi_{0}$ is the total volume concentration of the particles in the gel. For brevity, coefficients of proportionality are omitted in the right parts of (4) and (5).

According to the classical theory of elasticity of the polymer nets [49,50], like in the [42], we use the estimate:

$G_{d} \sim v$

(coefficient of proportionality is also omitted here).

Combining (4-6), we come to the following estimate:

$\Phi G_{d} \sim \varphi_{0} \psi_{d}^{2}$

The domains of the dense phase of the fibrin macromolecules, illustrated in Fig.8, appear due to the phase condensation, provoked by the particles, anchored by the fibrins. Thus, the energy $U$ of attraction between the fibrins is, roughly, proportional to the particle concentration on each macromolecule. This concentration is proportional to the ratio $\varphi_{0} / \psi_{0}$. By using standard results of the theory of the gas-liquid phase transitions, we can qualitatively present the binodal of this separation as it is shown in the Fig.9.

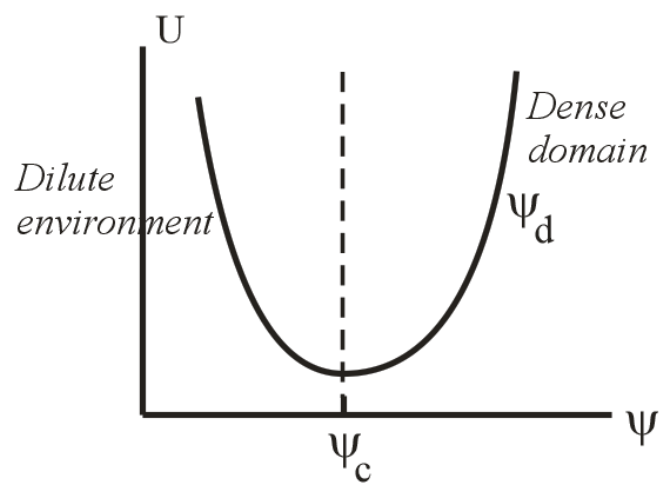

Fig.9. Illustration of the binodal of the phase transition in the system of the fibrins with anchored particles

Roughly we can describe this bimodal as:

$U-U_{c}=B\left(\psi-\psi_{c}\right)^{2}$

Here $U_{c}$ and $\psi_{c}$ are critical (from the viewpoint of the theory of the phase transitions) magnitudes of the corresponding parameters, $B$ is some parameter. To calculate $U_{c}, \psi_{c}$ and $B$, one needs to develop a theoretical model (similar to the Van der Waals one) of the condensation phase transition in the system of the flexible fibrin chains with the indirect interaction due to the 
adsorbed particles. This can be considered as a special problem which requires simultaneous experimental study of details of the interfibrin interaction. Here we will consider $B, U_{c}, \psi_{c}$ as empirical parameters. For maximal simplicity, in eq. (8), one can neglect $U_{c}$ as compared with the actual energy $U$. This simplification does not affect the final results; it just allows reducing the final results.

Neglecting $U_{c}$ in (8), by using the estimate $U \sim \varphi_{0}$, we get:

$\psi_{d}-\psi_{c} \sim \sqrt{\varphi_{0}}$

Combining (7) and (9), we come the following scaling relation:

$\Phi G_{d}=a \varphi_{0}^{2}+b \varphi_{0}^{3 / 2}+c \varphi_{0}$

Here $a, b, c$ are some parameters, which depend on the total concentration $\psi_{0}$ of the fibrins, details of electrostatic interaction between the particles and the polymer, as well as of the steric interaction between the fibrins. Independent determination of these parameters requires special experiments.

Taking into account the relation (2), one can come to the following expression for the total shear modulus of the system with the percolating domains of the dense phase:

$G \approx G_{0}+\Phi G_{d} \approx G_{0}+a \varphi_{0}^{2}+b \varphi_{0}^{3 / 2}+c \varphi_{0}$

The relation (11) qualitatively corresponds to the experimental curve. Comparison of the experimental and theoretical results is shown in Fig.10. The adjusted parameters $a, b, \mathrm{c}$ have been fitted as: $a=202731836 P a ; b=-11949999 P a ; c=269756 P a$

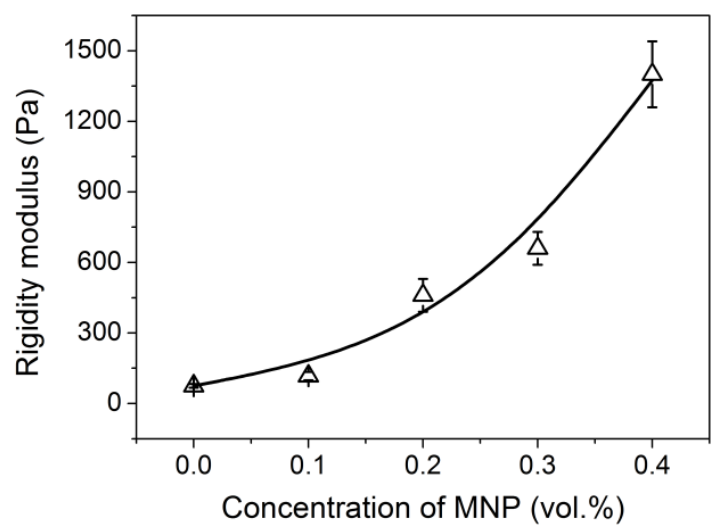

Figure 10. Comparison of the experimental (dots) and theoretical (line) results for the hydrogel cured under the field action. $G_{0}=75 P a$. Experimental dots are the same as in Fig.4.

It should be noted that parameters $a, b, c$ depend on the energy $U$ of the indirect attraction between the fibrins, on the critical concentration $\psi_{c}$ as well as on the parameters of elasticity of the fibrins in the domains. In their turn these magnitudes are determined by details of complicated molecular structure of the fibrin threads. Specification of $U, \psi_{c}, B$ as well as the fibrin elasticity can be a subject of separate studies. 


\section{Conclusion}

Our experiments show that the rigidity modulus of fibrin biogels is very sensitive to the presence of embedded magnetite particles. Very small addition (less than $0.5 \%$ in volume) of magnetite particles increases the rigidity modulus by more than 10 times; i.e., an increase about three orders of magnitude greater than the effect predicted by the classical theories of composite materials. This strong concentration dependence of the modulus can be explained on the basis of the concept that the embedded magnetite particles provoke phase condensation of the polymer and formation of dense knocks (domains), consisting of the fibrin macromolecules and the particles. If the biogel is cured without external magnetic field, the knocks are spherical-shaped. They serve as additional, to the chemical "ties", centers of the biogel cross-linkage; as a result the gel rigidity significantly increases. If the composite was gelled under the field action, the knocks get magnetized and, at the stage preceding the system complete curing, under the force of magnetic attraction, amalgamate into cylindrical domains, percolating the measuring cell. In this case, the macroscopic rigidity of the gelled composite is determined by the rigidity of the dense cylinders, which is much greater than that of the pure gel. Theoretical model, developed on the base of this concept, at least semiquantitatively, describes the experimental results.

\section{Acknowledgement.}

$\mathrm{AZ}$ is grateful to the program of the Ministry of Education and Science of the Russian Federation, projects 02.A03.21.0006; 3.1438.2017/4.6; 3.5214.2017/6.7 as well as to the Russian Fund of Basic Researches, project 18-08-00178. Financial support by projects FIS2013-41821-R (Plan Nacional de Investigación Científica, Desarrollo e Innovación Tecnológica, MINECO, Spain, co-funded by ERDF, European Union) and FIS2017-85954-R (Agencia Estatal de Investigación, AEI, Spain, co-funded by Fondo Europeo de Desarrollo Regional, ERDF, European Union) is also acknowledged.

\section{REFERENCES}

1.Bose, H., Rabindranath, R., Ehrlich, J.: Soft magnetorheological elastomers as new actuators for valves. J. Intell. Mater. Syst. Struct. 23 (9), 989-994 (2012).

2. Filipcsei G., Csetneki I., Szilagyi A., Zrınyi M.: Magnetic Field-Responsive Smart Polymer Composites, in Adv. Polym. Sci., 206, 137-189 ;Springer-Verlag Berlin Heidelberg 2007.

3. Boczkowska A., Awietjan S.: Tuning active magnetorheological elastomers for damping applications, Materials Science Forum, 636-637, 766-771 (2010);

4. Dyke S., Spencer B., Sain M., Carlson J.: Modeling and control of magnetorheological dampers for seismic response reduction, Smart Materials and Structures, 5(5), 565-575 (1996)

5. Occhiuzzi A., Spizzuoco M., Serino G.: Experimental analysis of magnetorheological dampers for structural control, Smart Materials and Structures, 12, 703-711 (2003). 
6. Carmona F., Mouney C.: Temperature-dependent resistivity and conduction mechanism in carbon particle-filled polymers, J. Mater. Science, 27(5), 1322-1326 (1992).

7. Feller J., Linossier I., Grohens Y., Conductive polymer composites: comparative study of poly(ester)-short carbon fibres and poly(epoxy)-short carbon fibres mechanical and electrical properties, Mat. Letters, 57(1) 64-71 (2002)

8. Bañobre-López M., Piñeiro-Redondo Y., de Santis R., Gloria A., Ambrosio L., Tampieri A., et al. : Poly(caprolactone) based magnetic scaffolds for bone tissue engineering,

J Appl Phys., 109 (7), 07B313 (2011)

9. Bock N., Riminucci A., Dionigi C., Russo A., Tampieri A., Landi E., et al.: A novel route in bone tissue engineering: Magnetic biomimetic scaffolds, Acta Biomater. 6 (3), 786-796 (2010) 10. Lin C., Metters A.: Hydrogels in controlled release formulations: Network design and mathematical modeling, Adv. Drug Delivery Reviews, 58 (12-13), 1379-1408 (2006).

11. Langer R.: New methods of drug delivery, Science, 249 (4976), 1527-1533 (1990).

12. Mitragotri S., Lahann J.: Physical approaches to biomaterial design, Nat. Mater., 8, 1521(2009).

13. Choi N.W., Cabodi M., Held B., Gleghorn J.P., Bonassar LJ, Stroock AD: Microfluidic scaffolds for tissue engineering, Nat. Mater., 6, 908-915 (2007).

14. Kurlyandskaya G.V., Fernández E., Safronov A.P., Svalov A.V., Beketov I., Beitia A.B., García-Arribas A., Blyakhman F. A.: Giant magnetoimpedance biosensor for ferrogel detection: Model system to evaluate properties of natural tissue, Appl. Phys. Letters , 106, 193702 (2015)

15. Thevenot J., Oliveira H., Sandre O., Lecommandoux S.: Magnetic responsive polymer composite materials, Chem. Soc. Reviews., 42(17), 7099-7116 (2013)

16. Hunt N. C., Grover L. M.: Cell encapsulation using biopolymer gels for regenerative medicine, Biotechnol. Letters, 32 (6),733-742 (2010)

17. Das B., Mandal M., Upadhyay A., Chattopadhyay P., Karak N.: Bio-based hyperbranched polyurethane $/ \mathrm{Fe}_{3} \mathrm{O}_{4}$ nanocomposites: smart antibacterial biomaterials for biomedical devices and implants, Biomed. Materials, 8 (3), 035003 (2013)

18. de Santis R., Gloria A., Russo T., d'Amora U., Zeppetelli S., Dionigi C.: A basic approach toward the development of nanocomposite magnetic scaffolds for advanced bone tissue engineering, J.Appl. Polym. Sci., 122(6), 3599-3605 (2011)

19. Gloria A., Russo R., d'Amora U., Zeppetelli S., d'Alessandro T., Sandri M., et al. : 
Magnetic poly( $\varepsilon$-caprolactone)/iron-doped hydroxyapatite nanocomposite substrates for advanced bone tissue engineering, J. R. Soc Interface, 10 (80), 20120833, (2013)

20. Hu S.H., Liu T.Y., Tsai C.H., Chen S.Y.: Preparation and characterization of magnetic ferroscaffolds for tissue engineering, J Magn Magn Materials, 310 (2), 2871- 2873 (2007) 21. Hu H., Jiang W., Lan F., Zeng X., Ma S., Wu Y., et al : Synergic effect of magnetic nanoparticles on the electrospun aligned superparamagnetic nanofibers as a potential tissue engineering scaffold, RSC, Adv., 3, 879-886 (2013)

22. Lai K., Jiang W., Tang J.Z., Wu Y., He B., Wang G., et al.:

Superparamagnetic nano-composite scaffolds for promoting bone cell proliferation and defect reparation without a magnetic field, RSC Adv. 2, 13007-13017 (2012)

23. Li Y., Huang G., Zhang X., Li B., Chen Y., Lu T., et al : Magnetic Hydrogels and Their Potential Biomedical Applications, Adv Funct Materials 23 (6), 660-672 (2013)

24. Panseri S., Cunha C., 'Alessandro T., Sandri M., Giavaresi G., Marcacci M., et al.: Intrinsically superparamagnetic Fe-hydroxyapatite nanoparticles positively influence osteoblastlike cell behaviour, J Nanobiotechnology, 10, 32 (2012)

25. Skaat H., Ziv-Polat O., Shahar A., Last D., Mardor Y., Margel S.: Magnetic Scaffolds Enriched with Bioactive Nanoparticles for Tissue Engineering, Adv Healthc Mater., 1 (2), 168$171(2012)$

26. Tampieri A., Landi E., Valentini F., Sandri M., d'Alessandro T., Dediu V., et al. : A conceptually new type of bio-hybrid scaffold for bone regeneration, Nanotechnology, 22 (1), $015104(2011)$

27. Tampieri A., d'Alessandro T., Sandri M., Sprio S., Landi E., Bertinetti L., et al. : Intrinsic magnetism and hyperthermia in bioactive Fe-doped hydroxyapatite, Acta Biomaterials, 8 (2), 843-851 (2012)

28. Zeng X.B., Hu H., Xie L.Q., Lan F., Jiang W., Wu Y., et al.: Magnetic responsive hydroxyapatite composite scaffolds construction for bone defect reparation,

Int. J. Nanomedicine, 7, 3365-3378 (2012)

29. Zeng X.B., Hu H., Xie L.Q., Lan F., Wu Y., Gu Z.W. :

Preparation and Properties of Supermagnetic?Calcium Phosphate Composite Scaffold, J Inorg Materials, 28 (1), 79-84 (2013) 
30. Zhu Y., Shang F., Li B., Dong Y., Liu Y., Lohe M.R., et al.: Magnetic mesoporous bioactive glass scaffolds: preparation, physicochemistry and biological properties, J Mater Chem B., 1 (9), 1279-1288 (2013)

31. Ziv-Polat O., Skaat H., Shahar A., Margel S.: Novel magnetic fibrin hydrogel scaffolds containing thrombin and growth factors conjugated iron oxide nanoparticles for tissue engineering, Int J Nanomedicine, 7, 1259-1274 (2012)

32. Singh R.K.,.Patel K.D, Lee J.H., Lee E.J., Kim J.H., Kim T.H., et al.: Potential of magnetic nanofiber scaffolds with mechanical and biological properties applicable for bone regeneration.

PLOS ONE, 9, e91584 (2014)

33. Lopez-Lopez M. T., Scionti G., Oliveira A.C., Duran J.D. G., Campos A., Alaminos M., Rodriges I.A.: Generation and Characterization of Novel Magnetic Field-Responsive Biomaterials, PLOS ONE, 10(7), e0133878 (2015)

34. Nicodemus G. D., Bryant S. J.: Cell encapsulation in biodegradable hydrogels for tissue engineering applications, Tissue Eng., Part B, 14(2), 149-65 (2008)

35. Ladet S., David L., Domard A.: Multi-membrane hydrogels, Nature, 452, 76-79 (2008).

36. Caló E., Khutoryanskiy V.V.: Biomedical applications of hydrogels: A review of patents and commercial products, Eur. Polym. J. 65, 252-267 (2015)

37. Sharmin et al. In: Injectable hydrogels for regenerative engineering. Imperial College Press, pp. 1-32 (2016).

38. Banobre-Lopez M., Pineiro-Redondo Y., de Santis R., Gloria A., Ambrosio L., Tampieri A., Dediu V., Rivas J.: Poly(caprolactone) based magnetic scaffolds for bone tissue engineering J. Appl. Physics, 109, 07B313 (2011).

39. Yun H. M., Ahn S. J., Park K. R., Kim M. J., Kim J. J., Jinc G. Z., Kim H. W., Kim E. C.: Magnetic nanocomposite scaffolds combined with static magnetic field in the stimulation of osteoblastic differentiation and bone formation, Biomaterials, 85, 88-98. (2016)

40. Rodriguez-Arco L., Rodriguez I. A., Carriel V., Bonhome-Espinosa A. B., Campos F., Kuzhir P., Duran J. D. G., Lopez-Lopez M. T.: Biocompatible magnetic core-shell nanocomposites for engineered magnetic tissues, Nanoscale, 8(15), 8138-8150 (2016).

41. Lopez-Lopez M. T., Rodriguez I. A., Rodriguez-Arco L., Carriel V., Bonhome-Espinosa A. B., Campos F., Zubarev A., Duran J. D. G.: Synthesis, characterization and in vivo evaluation of biocompatible ferrogels, J. Magn. Magn. Materials, 431, 110-114 (2017).

42. Bonhome-Espinosa A. B., Campos F., Rodriguez I. A., Carriel V., Marins J. A., Zubarev A., 
Duran J. D. G., Lopez-Lopez M. T.: Effect of particle concentration on the microstructural and macromechanical properties of biocompatible magnetic hydrogels, Soft Matter 13, 2928-2941 (2017)

43. Scionti G., Moral M., Toledano M., Osorio R., Durán J.D.G., Alaminos M., Campos A., to T. López-López M.T.: Effect of the hydration on the biomechanical properties in a fibrin-agarose tissue-like model, J. Biomed. Mater. Res. Part A, 102A, 2573-2582 (2014).

44. Alaminos M, Sanchez-Quevedo MC, Munoz-Avila JI, Serrano D, Medialdea S, Carreras I, et al.: Construction of a complete rabbit cornea substitute using a fibrin-agarose scaffold. Invest Ophthalmol Vis. Science, 47, 3311-3317 (2006)

45. Bychkova A. V., Sorokina O. N., Kovarski A. L., Shapiro A. B., Leonova V. B. and Rozenfeld M. A.: Interaction of fibrinogen with magnetite nanoparticles,

Biophysics, 55 (4), 544-549, (2010)

46. Cote H. C. F., Lord S. T., Pratt K. P. $\gamma$-Chain Dysfibrinogenemias: Molecular StructureFunction Relationships of Naturally Occurring Mutations in the $\gamma$-Chain of Human Fibrinogen: Blood, 92(7), 2195-2212 (1998)

47. Zeliszewska P., Bratek-Skicki A., Adamczyk Z.,Ciesla M.: Human Fibrinogen Adsorption on Positively Charged Latex Particles, Langmuir, 30(37), 11165- 11174 (2014)

48. Pitaevskii L. P., Lifshits E. M., Physical Kinetics, Butterworth-Heinemann, (1999) 49. Rubistein M., Colby R. H., Polymer Physics, Oxford University, New York, NY, (2003) 50. A. Grosberg and A. Khokhlov, Statistical Physics of Macromolecules, Springer, Berlin, (1994).

51. R. M. Christensen, Mechanics of Composite Materials, Krieger Publishing Company, Malabar, (1991). 